\title{
An evaluation of the relationship between the range of mandibular opening and the condyle positions in functional panoramic radiographs
}

\section{Ocena związku między zakresem opuszczania żuchwy a pozycjami wyrostków kłykciowych na czynnościowych zdjęciach pantomograficznych}

\author{
Jolanta E. Loster ${ }^{1, A-F}$, Magdalena Groch ${ }^{1, A, C, D, F}$, Aneta Wieczorek1,A-C,E,F, Małgorzata Muzalewska ${ }^{2, B, C, F}$, Wojciech Skarka ${ }^{2, A-C, E, F}$ \\ 1 Department of Dental Prosthetics, Institute of Dentistry, Jagiellonian University, Kraków, Poland \\ ${ }^{2}$ Institute of Fundamentals of Machinery Design, Faculty of Mechanical Engineering, Silesian University of Technology, Gliwice, Poland \\ A - research concept and design; $\mathrm{B}$ - collection and/or assembly of data; $\mathrm{C}$ - data analysis and interpretation; \\ $\mathrm{D}$ - writing the article; $\mathrm{E}$ - critical revision of the article; $\mathrm{F}$ - final approval of the article
}

Address for correspondence

Jolanta E. Loster

E-mail: jolanta.loster@uj.edu.pl

Funding sources

None declared

Conflict of interest

None declared

Received on August 17, 2017

Reviewed on October 13, 2017

Accepted on 0ctober 26, 2017

D0I

10.17219/dmp/79255

Copyright

○ 2017 by Wroclaw Medical University

and Polish Dental Society

This is an article distributed under the terms of the

Creative Commons Attribution Non-Commercial License

(http://creativecommons.org/licenses/by-nc-nd/4.0/)

\begin{abstract}
Background. Radiographic diagnostics in dentistry is one additional examination which facilitates an accurate clinical diagnosis and, as a result, the initiation of appropriate treatment. Despite various limitations, functional panoramic radiograph (OPG) images of the temporomandibular joints (TMJ) provide a great deal of valuable information and seem to be the first-choice modality in the diagnosis of temporomandibular disorders.
\end{abstract}

Objectives. The aim of this study was to evaluate any relationship between the range of mouth opening and the condyle positions during this movement, on the basis of functional OPG images.

Material and methods. To evaluate the distance between the condyle positions, 10 functional OPG images of the TMJ were used. The relationship between the measurements taken during clinical examination of the patients and the measurements obtained from functional OPG images were evaluated. The research hypothesis assumed that there was a positive correlation between the clinical range of mouth opening and the measurement of condyle movement from a centric occlusion position to its maximum opening.

Results. The analysis of measurements showed no statistical correlation between the distances between the condyle positions obtained from radiographic images and clinical measurements of the range of mouth opening.

Conclusions. In young, healthy patients without clinical symptoms of TMJ dysfunction, functional OPG images of the joint should not be compared with the range of mandibular opening.

Key words: temporomandibular joint, X-ray images, condylar pathway

Słowa kluczowe: staw skroniowo-żuchwowy, zdjęcia rentgenowskie, droga stawowa 
Radiographic diagnostics in dentistry is one additional examination which facilitates accurate clinical diagnosis and, as a result, the initiation of appropriate treatment. In recent years, the quality of the images produced has improved, and the options of using X-rays for human body imaging have expanded significantly along with a simultaneous reduction in the ionising radiation dose necessary to obtain images of clinically acceptable quality. ${ }^{1-3}$

Clinical and radiological diagnostics of temporomandibular joints (TMJ) is extremely complex. ${ }^{4}$ Symptoms reported by patients are often seemingly unrelated to the joints themselves and analysis of the radiological images of the TMJ may present difficulties due to their anatomical complexity. ${ }^{1,5}$ Therefore, appropriately selecting the type of additional examinations and interpreting their results seem to be even more important. One should keep in mind, however, that regardless of the technique and view in which images are captured, X-ray images are meant to complement clinical and instrumental examinations and should only be interpreted with their results in mind. ${ }^{6}$

Currently, a range of imaging modalities to visualise TMJ structures are available, such as magnetic resonance imaging (MRI), cone beam computed tomography (CBCT), computed tomography $(\mathrm{CT})$, and panoramic radiograph (OPG). A distinctive feature of MRI examination is that it allows soft-tissue imaging, which enables one to evaluate the position of the articular disk and the surrounding soft tissues in TMJ disorders. As three-dimensional imaging methods, CBCT and CT examinations provide a visualisation of bone structures. The CT method exposes patients to the highest ionising radiation dose of all TMJ imaging methods. MRI is a detailed examination considered in the literature to be the 'gold standard' in diagnostics of TMJ abnormalities; however, it is not commonly used in everyday dental practice due to economic factors. ${ }^{7}$ On the other hand, in order to protect the patient, each radiological examination should be supported by appropriate indications, and the number of such procedures should be limited to the bare minimum. One of the basic plain examinations of the TMJ is an OPG, commonly used and widely available in dental treatment. ${ }^{8}$ Admittedly, the image obtained is a flat reflection of the curved surface of the maxilla and a composite image of the tissues located in the X-ray's path; however, it provides a sufficiently accurate visualisation of the bone structures around the TMJ. ${ }^{8,9}$ A functional OPG is a modification of an OPG examination and is performed in the following positions: habitual occlusion (maximum contact between the teeth of opposing arches) and maximum opening (maximum opening of the mandible in relation to the maxilla), each separately for the right and left sides (a separate image for each TMJ). As a result, 4 images on one film are obtained. Analysing such images enables one to evaluate the bone components of both joints and, indirectly, the articular disk, and provides information on the condylar range of motion between the position of habitual occlu- sion (maximum intercuspation) and maximum opening of the mandible in relation to the maxilla. When evaluating X-ray images, the anatomy of the left and right joints is compared. This allows both the exclusion of any potential deviations from normal geometry, and at the same time control over the relationship between the 2 joints during their function, i.e., at the beginning and at the end of opening movement. The unquestionable advantages of the examination also include its ready availability, low costs of performance, and non-invasiveness. One of the major weaknesses of OPG images is their magnification, which results from the radiographic technique applied. Although the magnification factor is fixed, differences in magnification occur even within the same radiograph. Due to the layered nature of this examination, shadows of other anatomical structures are superimposed over the captured image, which make interpretation of the image difficult. Despite these limitations, functional OPG images of the TMJ provide a great deal of valuable information and seem to be the modality of choice in the diagnosis of temporomandibular disorders. There are no publications available that discuss the interpretation of these images, hence the need to undertake the study below. ${ }^{10,11}$

The aim of this paper was to evaluate any relationship between the range of mandibular opening and the condyle positions during this movement on the basis of functional OPG images.

\section{Material and methods}

To evaluate the distance between the condyle positions, functional OPG images of the TMJ captured in intercuspation and maximum mandibular opening positions were used. The images were obtained from 10 randomly selected 18-year-old volunteers (from a group of 260 individuals) taking part in a project supported by the Polish Ministry of Science and Higher Education no. N N403 589138. ${ }^{12}$

All images were obtained with the same ProMax ${ }^{\circledR}$ scanner (Planmeca, Helsinki, Finland 2005), by the same radiology technician, using the same radiation parameters in current $(14.0 \mathrm{~mA})$ and voltage $(70 \mathrm{kV}) .{ }^{7}$ A stomatognathic system examination was performed in all project participants by one calibrated examiner in accordance with the Research Diagnostic Criteria for Temporomandibular Disorders (RDC/TMD) ${ }^{13}$ Presenting individuals were informed of the project's objective and that they can withdraw at any time without giving a reason. Volunteers provided written informed consent to participate in the study. The study was approved by the Bioethical Committee of the Jagiellonian University under the number $\mathrm{KBET} / 89 / \mathrm{B} / 2009$ and was conducted in accordance with the principles laid down in the Declaration of Helsinki and Good Clinical Practice (GCP). Ten patients were selected randomly from the group of 260 examined individuals, regardless of gender. Data regarding the maximum range 
of the mandible opening (pain-free opening), midline coincidence between upper and lower incisors in a centric occlusion position, range of vertical incisal distance, and curve of movement path were obtained from the RDC/ TMD Axis I Clinical Examination Form after clinical examination was done by a single calibrated specialist in prosthodontics (MG). ${ }^{12}$ The study inclusion criteria included the following: no symptoms of temporomandibular disorders according to RDC/TMD, no midline deviation, and a straight line of the mandibular opening.

Using data regarding the range of opening of the mandible collected during a clinical examination performed in accordance with RDC/TMD Axis I, further analysis was carried out. Values for vertical range of motion (pain free opening) obtained from the form were added to the overbite distance, and thus the actual opening range of motion was obtained.

\section{Image evaluation methodology}

Each radiographic image was analysed by one examiner (MM). Figure 1 shows all elements drawn. On images made in the maximum intercuspation position (designated as I), pathways were drawn according to the shape of the cusp and the articular fossa (I.1), and the condylar head and ramus of the mandible (I.2). ${ }^{14}$ Next, the highest point of the articular fossa (I.1.a) and the highest point of the condylar head (I.2.b) were marked on the pathways drawn. The measurements consisted of measuring the distance between the highest point of the condylar head and the highest point of the articular fossa (connecting points I.1.a and I.2.b), and determining the angle between the tangent passing through the penultimate point on the curve of the mandibular ramus and the line parallel to the $\mathrm{X}$-axis (I.4).

The next step of the analysis was to transfer the pathways, along with the points marked on them, to images

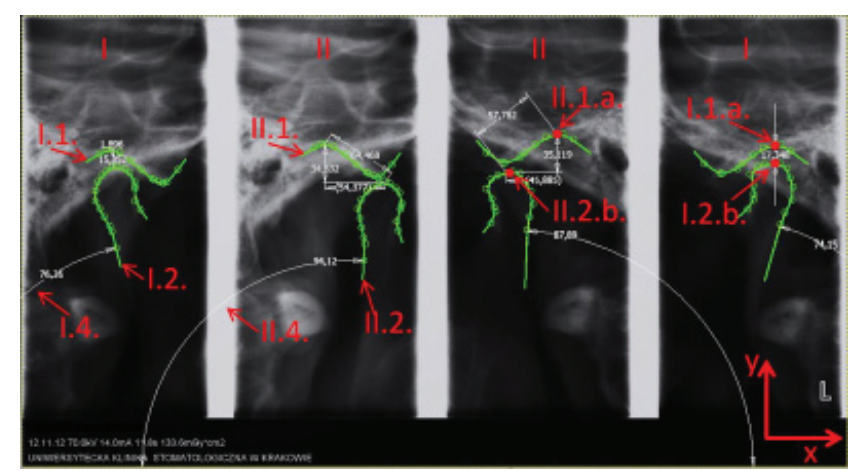

Fig. 1. An example image with points, pathways, and measurements drawn. I images - condylar heads in maximum intercuspation; II images - heads of the mandible in maximum opening. $L$ - patient's left side. I.1 - the curve corresponding to the curvature of the cusp and articular fossa; 1.2 - the curve corresponding to the condylar head and mandibular ramus; I.1.a - the highest point of the articular fossa; I.2.b - the highest point of the condylar head; I.4 - the angle formed between the tangent passing through the penultimate point on the curve of the mandibular ramus and the line parallel to the $x$-axis. Analogous designations for II images showing the TMJ in the maximum opening position (designated as II). Pathways corresponding to the picture of the cusp and the articular fossa along with its highest point (II.1.a), and the picture of condylar head with its highest point (II.2.b), were created. The distances between points II.1. $\mathrm{a}$ and II.2.b (the distance between points $\mathrm{a}$ and $\mathrm{b}$ and the shift towards the $\mathrm{x}$-axis [horizontal] and towards the $y$-axis [vertical]) were measured, and the angle between the tangent passing through the penultimate point on the curve of the mandibular ramus and the line parallel to the $\mathrm{x}$-axis (II.4) was determined.

When analysing individual measurements, the right and left sides in each patient were compared to the right and left sides in all patients. The relationship between the measurements taken during clinical examination of the patients (measurements between the incisal edges of the lower and upper dental arches in the maximum opening position, increased by the range of the overbite distance [actual range of opening]) and those obtained from X-ray images was evaluated. The research hypothesis assumed that there was a positive correlation between the clinical range of opening of the mandible and the measurement of the condylar head movement from centric occlusion to maximum opening.

For statistical analysis of the results obtained, Student's $\mathrm{t}$-test with a significance level of $\mathrm{p}=0.05$ was applied.

\section{Results}

All 10 of the radiographic images which were randomly selected from a group of 260 individuals (including 192 women and 68 men) and then evaluated were images received from women. Table 1 shows the measurements obtained from the analysis of OPG images. No direct impact of the segment lengths from points II.1.a to II.2.b on the values of the range of opening of the mandible which were received during the clinical evaluation was identified (Table 1). The analysis of measurements showed no statistical correlation between the distances from the condyle positions obtained from radiographic images and clinical measurements of the range of opening of the mandible (Fig. 2, 3).

\section{Discussion}

Analysis of the results obtained suggests a lack of correlation between the clinical measurements of the range of opening of the mandible and the range of motion of the mandibular heads when their position is changed from maximum intercuspation to maximum opening, shown using radiological imaging in the form of functional OPG images.

This may be due to the characteristics of OPG imagery, which is a composite picture with anatomical structures 
Table 1. Mean results obtained from the analysis

\begin{tabular}{|c|c|c|c|c|c|c|c|}
\hline Image points & Unit & $\begin{array}{l}\text { Mean } \\
\text { - right side }\end{array}$ & $\begin{array}{l}\text { Standard } \\
\text { deviation }\end{array}$ & $\begin{array}{l}\text { Mean } \\
\text { - left side }\end{array}$ & $\begin{array}{l}\text { Standard } \\
\text { deviation }\end{array}$ & Difference & $\begin{array}{l}\text { Standard deviation, } \\
\text { right side and left sides }\end{array}$ \\
\hline I.1.a-I.2.b distance & {$[\mathrm{mm}]$} & 2.01 & 0.6 & 1.89 & 0.88 & 0.12 & 0.08 \\
\hline 1.4. angle & {$\left[{ }^{\circ}\right]$} & 49.09 & 6.62 & 47.66 & 7.67 & 1.43 & 1.01 \\
\hline II.1.a-II.2.b distance & {$[\mathrm{mm}]$} & 8.95 & 1.32 & 9.46 & 1.72 & 0.51 & 0.36 \\
\hline II.1.a-II.2.b distance on the $x$-axis & {$[\mathrm{mm}]$} & 7.84 & 1.24 & 8.47 & 1.92 & 0.63 & 0.45 \\
\hline II.1.a-II.2.b distance on the $y$-axis & {$[\mathrm{mm}]$} & 4.26 & 0.96 & 3.96 & 1.19 & 0.3 & 0.21 \\
\hline II.4 angle & {$\left[{ }^{\circ}\right]$} & 65.92 & 8.01 & 64.83 & 5.81 & 1.10 & 0.78 \\
\hline Difference between 1.4 and II.4 angles & {$\left[{ }^{\circ}\right]$} & 16.84 & 3.49 & 17.17 & 3.72 & 0.33 & 0.23 \\
\hline
\end{tabular}

| - condylar heads in maximum intercuspation, || - heads of the mandible in maximum opening.

I.1.a the highest point of the articular fossa, I.2.b the highest point of the condylar head, I.4 angle formed between the tangent passing through a penultimate point on the curve of the ramus of the mandible and the line parallel to the X-axis. Analogous designations for images II.

$\mathrm{mm}$ - millimetre, ${ }^{\circ}$ - degree

superimposed. This issue was particularly visible when the angular values of a change in position of the mandibular ramus were determined. On the other hand, the methodology of performing such an examination seems to have no impact on the results obtained, as the examination is performed at almost the same time for the right and left sides, and the position of the anatomical components of both sides is recorded during the same (one) movement performed by the patient. In view of such results, the question is whether the use of OPG images of the TMJ to determine the size of the joint space gives accurate results? Some authors use such images and measure the size of the joint space on a baseline image at the beginning of prosthetic treatment, and then compare the measurements to a post-prosthetic treatment image. In this case, the measurements of the three-dimensional joint's components are made on a two-dimensional image. The issue of the repeatability of these images is also interesting; however, it was not the objective of this study.

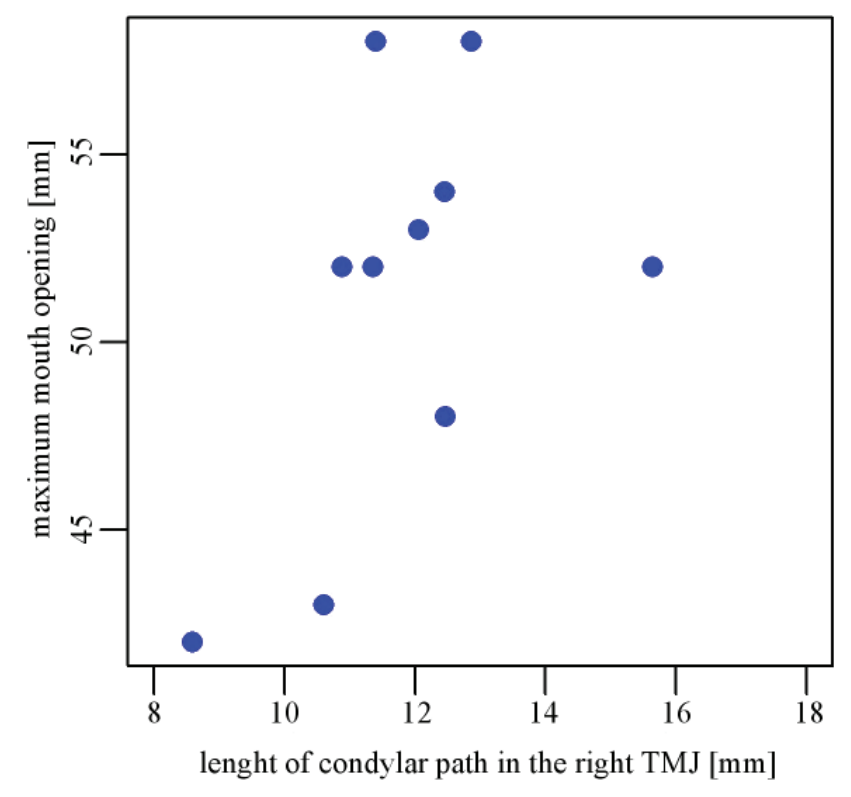

Fig. 2. The correlation between the maximum opening and length of the condylar pathway in the right TMJ
Studies were found in the literature whose objective was to evaluate the impact of a change in a patient's head position during a panoramic radiography examination on vertical measurement results in the ramus and condylar area of the mandible. The greatest asymmetry of right and left sides with differences of up to $6 \%$ was noted by the authors when the head was tilted down. ${ }^{15}$

The results obtained and the lack of expected correlation may also be due to the fact that the group of patients studied was small. Additionally, on the one hand, the young age of the examined patients ensured homogeneity within the group; on the other hand, it was not possible to compare with other age groups.

The use of computed tomography (CT) or magnetic resonance imaging (MRI) scans for measurements of the lengths and widths of particular structures of the TMJ seems to be the most appropriate. ${ }^{16}$ Studies comparing TMJ anatomical preparations to their CT and MRI scans showed no statistical differences between the measure-

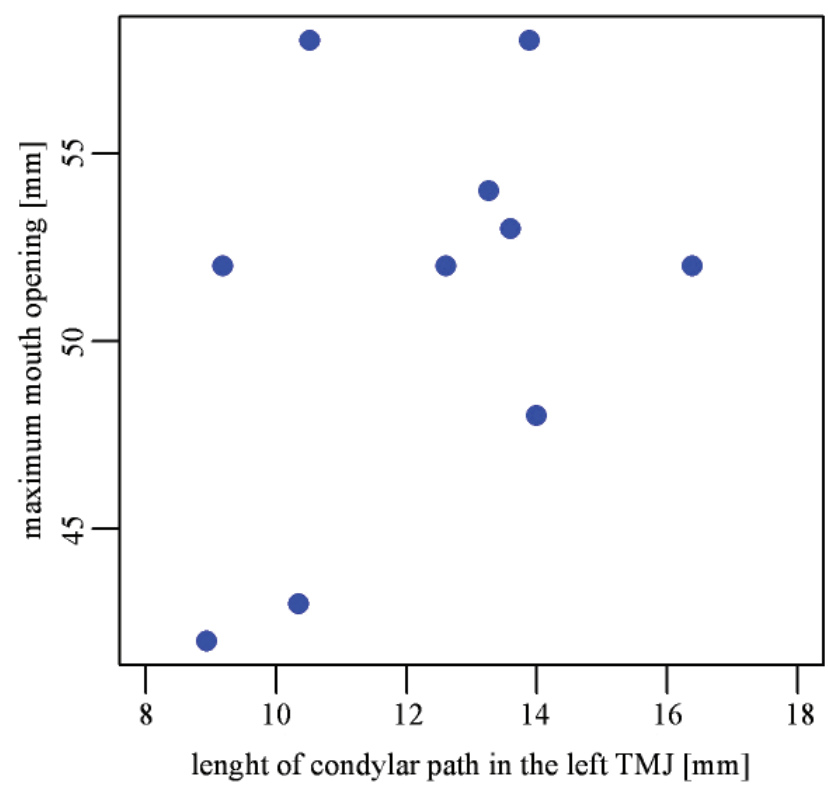

Fig. 3. The correlation between the maximum opening and length of the condylar pathway in the left TMJ 
ments of respective points within bone structures. ${ }^{17}$ Unfortunately, the availability of these imaging methods in everyday dental practice is limited.

Currently, cone-beam computed tomography (CBCT) is becoming increasingly popular, in diagnostics of TMJ disorders as well. It enables one to view the joint's hard tissues in 3 planes - sagittal, coronal, and axial - and to perform their three-dimensional reconstruction, which provides additional information on the anatomy of the joint and the position of the mandibular head in the glenoid fossa. ${ }^{18}$ Compared to a CT examination, CBCT offers higher resolution and requires a radiation dose even 20-30 times lower. It is also possible to select the scanning region size. ${ }^{19}$

When interpreting TMJ images, one should also keep in mind that according to the definition of centric relation, a central position of the condyle in the articular fossa is not the most optimal and stable position, as in individuals with no symptoms of masticatory functional disorders, the mandibular head may take various positions in the articular fossa. ${ }^{20}$ From a clinical perspective, missing pathological symptoms on the part of the masticatory system are the most important, rather than the position of condylar head in the articular fossa of the TMJ on the radiographic image. ${ }^{4}$

\section{Conclusions}

In young, healthy patients without clinical symptoms of TMJ dysfunction the functional OPG images should not be compared with the range of the mandibular opening. Further studies in this area are recommended, taking into account the selection of cases for analysis in terms of the presence of potential symptoms of masticatory functional disorders.

\section{References}

1. Magnusson T, Karlsson C. Clinical impact of radiological examinations of patients with suspected temporomandibular disorders. Swed Dent J. 2002;26:67-74.

2. Larheim TA. Current trends in temporomandibular joint imaging. Oral Surg Oral Med Oral Pathol Oral Radiol Endod. 1995;80:555-576.

3. Loster JE, Williams S, Wieczorek A, Loster BW. The polish face in profile: A cephalometric baseline study. Head Face Med. 2015;11:5.

4. Osiewicz MA, Lobbezoo F, Loster BW, Loster JE, Manfredini D. Frequency of temporomandibular disorders diagnoses based on RDC/TMD in a Polish patient population. Cranio. 2017;1-7.

5. Winocur E, Reiter S, Krichmer M, Kaffe I. Classifying degenerative joint disease by the RDC/TMD and by panoramic imaging: A retrospective analysis. J Oral Rehabil. 2010;37:171-177.

6. Wieczorek A, Loster J, Majewski S. Assessment of suitability of orthopantomographs in dental diagnostics of temporomandibular joints. J Stoma. 2012;65:845-854

7. Dworkin SF, LeResche L. Research diagnostic criteria for temporomandibular disorders: Review, criteria, examinations and specifications, critique. J Craniomandib Disord. 1992;6:301-355.

8. Loster JE, Wieczorek A, Ryniewicz WI. Condylar guidance angles obtained from panoramic radiographic images: An evaluation of their reproducibility. Dent Med Probl. 2017;54:35-40.
9. Boeddinghaus R, Whyte A. Current concepts in maxillofacial imaging. Eur J Radiol. 2008;66:396-418.

10. Fryback DG, Thornbury JR. The efficacy of diagnostic imaging. Med Decis Making. 1991;11:88-94.

11. Honey OB, Scarfe WC, Hilgers MJ, et al. Accuracy of cone-beam computed tomography imaging of the temporomandibular joint: Comparisons with panoramic radiology and linear tomography. Am J Orthod Dentofacial Orthop. 2007;132:429-438.

12. Loster JE, Osiewicz MA, Groch M, Ryniewicz W, Wieczorek A. The prevalence of TMD in polish young adults. J Prosthodont. 2017;26:284-288.

13. Osiewicz MA, Lobbezoo F, Loster BW, Wilkosz M, Naeije M, Ohrbach R. Research diagnostic criteria for temporomandibular disorders (RDC/TMD) - the polish version of a dual-axis system for the diagnosis of TMD. RDC/TMD form. J Stoma. 2013;66:576-649.

14. Ahn SJ, Kim TW, Lee DY, Nahm DS. Evaluation of internal derangement of the temporomandibular joint by panoramic radiographs compared with magnetic resonance imaging. Am J Orthod Dentofacial Orthop. 2006;129:479-485.

15. Sadat-Khonsari R, Fenske C, Behfar L, Bauss O. Panoramic radiography: Effects of head alignment on the vertical dimension of the mandibular ramus and condyle region. Eur J Orthod. 2012;34:164-169.

16. Raustia AM, Pyhtinen J. Morphology of the condyles and mandibular fossa as seen by computed tomography. J Prosthet Dent. 1990;63:77-82.

17. Gedrange T, Gredes T, Hietschold V, et al. Comparison of reference points in different methods of temporomandibular joint imaging. Adv Med Sci. 2012;57:157-162.

18. Hilgers ML, Scarfe WC, Scheetz JP, Farman AG. Accuracy of linear temporomandibular joint measurements with cone beam computed tomography and digital cephalometric radiography. Am J Orthod Dentofacial Orthop. 2005;128:803-811.

19. Barghan S, Tetradis S, Mallya S. Application of cone beam computed tomography for assessment of the temporomandibular joints. Aust Dent J. 2012;57(Suppl. 1):109-118.

20. The glossary of prosthodontic terms. J Prosthet Dent. 2005;94:10-92. 
